\title{
A rare case of gastric-type mucinous endocervical adenocarcinoma in a 59-year-old woman
}

\author{
Francesk Mulita, Fotios Iliopoulos, Ioannis Kehagias \\ Department of Surgery, General University Hospital of Patras, Patras, Greece
}

\begin{abstract}
Introduction: Endocervical adenocarcinoma represents 20-25\% of the histological types of cervical carcinoma. Gastric-type mucinous adenocarcinoma of the cervix is a rare type of cancer with aggressive behavior. This type of malignancy is not related to high-risk human papillomavirus (HPV).

Case report: We report a 59-year-old postmenopausal woman complaining of vaginal bleeding and pelvic pain. Histological analysis of punch biopsy and endocervical curettage revealed possible endocervical mucinous adenocarcinoma, while magnetic resonance imaging (MRI) revealed a $10 \times 8 \mathrm{~cm}$ sized cervical mass. According to oncologists, the tumor was inoperable, so the patient received 6 cycles of chemotherapeutic agents with carboplatin, paclitaxel and bevacizumab from December 2019 to March 2020. However, chemotherapy had very poor results in this patient, because the mass was increased to $24 \mathrm{~cm}$ in a new MRI performed after the end of chemotherapy. Finally, the patient underwent radical surgery. On histopathological examination, the surgical margin was all free from everywhere and the mass was confirmed as a gastric-type mucinous adenocarcinoma of the cervix with low differentiation.

Conclusion: It is very important to have an early diagnosis of gastric-type mucinous adenocarcinoma of the cervix, as this tumor is poorly symptomatic and very aggressive. When chemotherapy is not effective, radical surgery may be a solution for better survival.
\end{abstract}

Key words: endocervical, adenocarcinoma, mucinous, gastric-type, HPV.

\section{Introduction}

Endocervical adenocarcinoma represents $20-25 \%$ of the histological types of cervical carcinoma, with increasing incidence in recent years [1, 2]. Less frequently, histological subtypes are serous, clear cell, mesonephric and mucinous (intestinal, gastric or signet ring cells) adenocarcinoma [3]. According to the 2014 Classification of Tumours of Female Reproductive Organs by the WHO, gastric-type adenocarcinoma is included as a variant of mucinous endocervical adenocarcinomas [3]. Endocervical gastric-type adenocarcinoma, like other cervical adenocarcinomas, is difficult to diagnose and is the main subtype not associated with high-risk human papillomavirus (HPV) [1]. Early recognition and diagnosis of this malignancy is very important. In this report, we discuss a case of large gastric-type mucinous adenocarcinoma, which was diagnosed in a 59-year-old female patient.

\section{Case report}

A 59-year-old postmenopausal woman complaining of vaginal bleeding and pelvic pain presented to the gynecological department in May 2019. The woman had a significant past medical history of deep vein thrombosis (DVT) and her past surgical history included appendectomy 45 years ago. Her family history is negative for malignant ovarian and breast cancer in first-degree relatives. Previous screening for cervical cancer had not been performed.

Clinical and gynecological examination revealed no abnormalities, while transvaginal ultrasonography (TVS) was performed and revealed a cystic lesion $10 \times 7 \mathrm{~cm}$ in diameter on the left ovary and a cystic lesion $1 \times 1 \mathrm{~cm}$ in diameter on the right ovary. She underwent colposcopic examination followed by punch biopsy and endocervical curettage. Histological analysis of punch biopsy and endocervical curettage revealed possible endocervical mucinous adenocarcinoma. The differential diagnosis included either endocervical gastric-type mucinous adenocarcinoma, or metastatic mucinous colorectal or ovarian adenocarcinoma. Pelvis magnetic resonance imaging $(\mathrm{MRI})$ revealed a $10 \times 8 \mathrm{~cm}$ sized cervical mass (Fig. 1) and enlarged retroperitoneal lymph nodes.

In November 2019, the Council of Gynecologic Oncology decided that the tumor was inoperable, because it had a large diameter and there was a suspicion that 

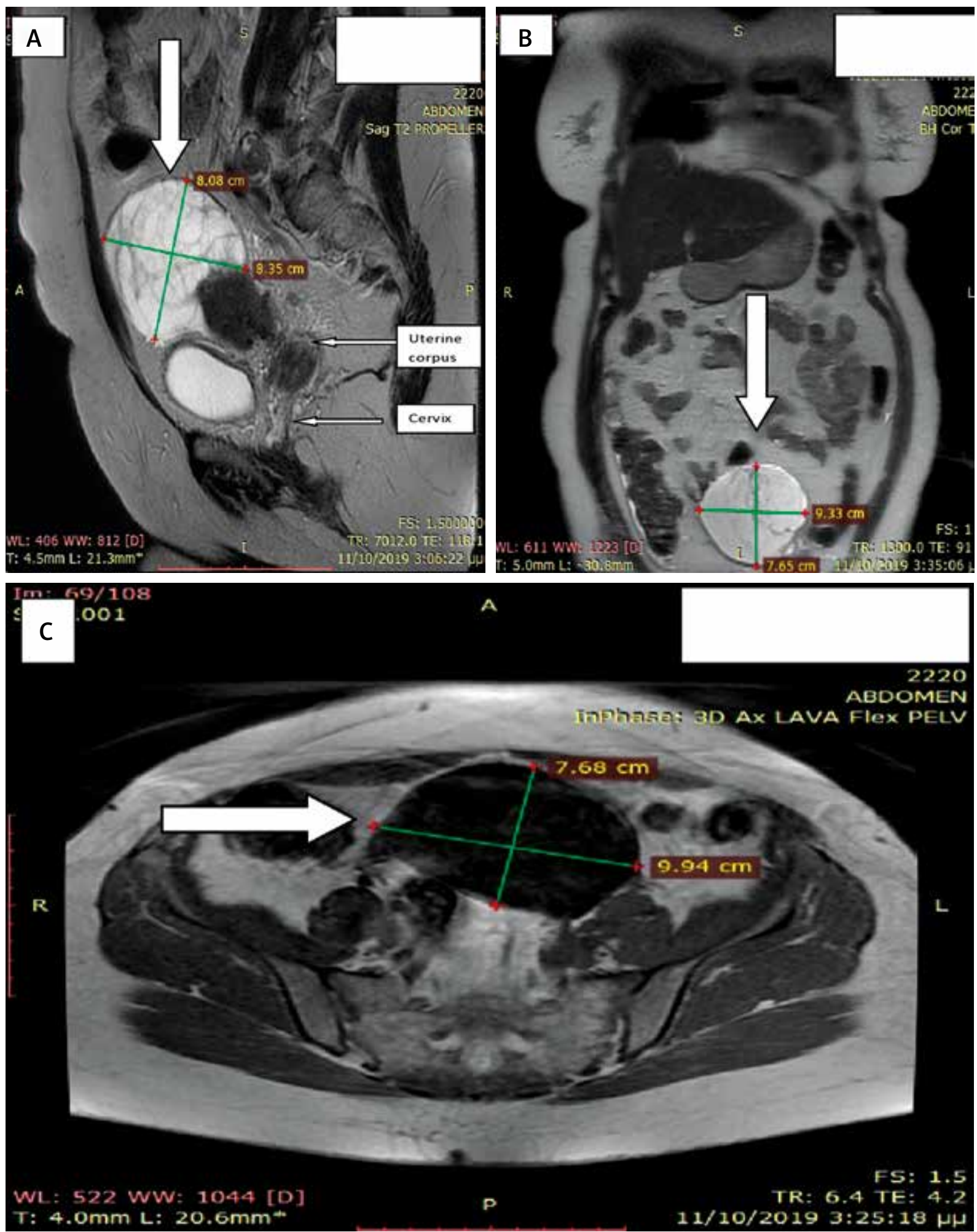

Fig. 1. Pelvis magnetic resonance imaging revealed a $10 \times 8 \mathrm{~cm}$ cervical mass

the tumor had spread to the bladder. The patient received 6 cycles of chemotherapeutic agents with carboplatin, paclitaxel and bevacizumab from December 2019 to March 2020. Although there was a decrease in the level of all tumor markers after chemotherapy, a new pelvis MRI showed enlargement of the cervical mass, which measured $24 \times 21 \times 12 \mathrm{~cm}$.
The patient underwent total abdominal hysterectomy with bilateral salpingo-oophorectomy, bilateral pelvic and aortic lymph node dissection and complete omentectomy. Intraoperatively the tumor was determined to be about $30 \mathrm{~cm}$ (Fig. 2 A, B) and had invaded part of the bladder, as well as the sigmoid colon, so a left hemicolectomy and partial cystectomy was also performed. Her final his- 


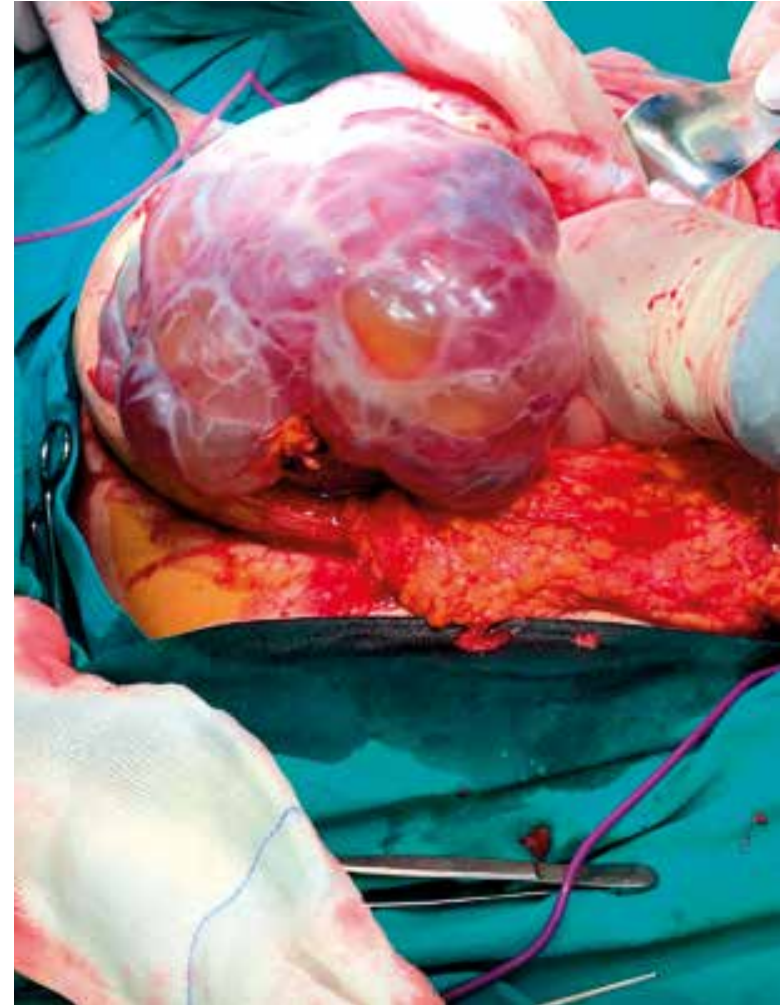

Fig. 2. Intraoperatively the tumor was determined to be about $30 \mathrm{~cm}$

topathology report indicated a gastric-type mucinous adenocarcinoma of the cervix with low differentiation. The surgical margin was all free from everywhere. The patient was discharged and referred to the oncology department for further management. Three months after surgery, a CT scan of the abdomen and pelvis was performed with the absence of the uterus and ovaries, without lymph node enlargement or other changes. Further management was continued by the oncology team for possible adjuvant chemoradiation therapy after radical surgery.

\section{Discussion}

Cervical cancer is one of the most common malignancies that arise from the female genital tract, being the third most frequent malignancy to affect women worldwide [4]. Carcinomas pertaining to the uterine cervix can be grossly divided into two categories: squamous cell carcinoma (SCC) that affects the ectocervix and adenocarcinoma (AC) which forms in the endocervix, the part of the cervix that connects the internal with the external cervical os.

AC is held accountable for $10-25 \%$ of all uterine cervical carcinomas in developed countries. It exhibits a gradually increasing incidence, following the declining frequency of SCC due to improved screening in the general population. The main pathogenetic factor for $80 \%$ of ACs is considered to be infections by high risk HPV subtypes, mainly types 16,18 and 45 [5].

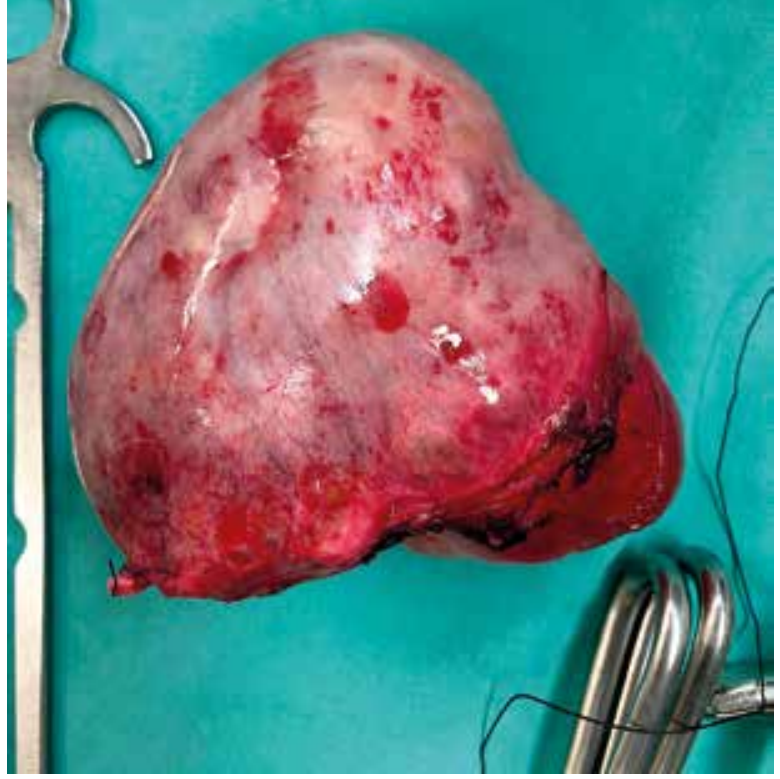

Fig. 3. Surgical specimen of the tumor

Although until recently ACs of the endocervix were classified according to the WHO classification, this has changed in favor of the International Endocervical Adenocarcinoma Criteria and Classification (IECC) system, which categorizes them based on morphological features linked to etiology. According to the IECC, endocervical ACs are distinguished into two broad groups: those that are associated with HPV, which are cytologically recognized by the presence of luminal mitoses and apoptosis (HPVA), and those not associated with HPV infection (NHPVA) [6]. The HPVA group includes the usual type $A C$, the mucinous $A C$ and its subtypes (the not otherwise specified [NOS], the intestinal and the signet ring cell type), the villoglandular type and the invasive stratified mucin-producing carcinoma (iSMILE). The HPVA group comprises the gastric type AC, the mesonephric, the serous and the clear cell carcinoma, the endometrioid AC and the NOS AC [7].

The most common HPVA carcinoma is the usual type (70-80\% of ACs), and the most common NHPVA type is the gastric type (10-11.7\%). Mucinous HPVAs of various subtypes (mucinous NOS, iSMILE, and intestinal type) are less frequent, representing $8.6 \%$ of all ACs. Clear cell carcinoma is the second most common NHPVA (3-3.4\%), whereas the following types are considered as rare: endometrioid (1.1\%), serous (0.5\%) and mesonephric (0.3-0.5\%) [8].

In our case, the patient's HPV DNA test was negative and immunohistochemical stains were negative for $p 16$, p40, p63, CK 5/6, CK 20, vimentin, Gata-3, TTF-1, napsin $A$ and positive for CK 7, CDX2, p53 and DPC4. According to the literature, not only HPV DNA, but also p16 protein is usually negative in gastric-type mucinous ACs of the cervix [9]. In addition, carcinoembryonic antigen (CEA) can be used as a good marker for differential diagnosis be- 
tween clear cell carcinoma and gastric-type ACs, because CEA is usually negative in clear cell carcinoma, while it is elevated in patients with gastric-type mucinous ACs of the cervix [10]. Our patient had an elevated level of CEA.

There is also a case of a 59-year-old woman with no abnormalities noted in the clinical evaluation. Her histological analysis from biopsies revealed cervical intraepithelial neoplasia (CIN) 1 and extensive lobular endocervical glandular hyperplasia (LEGH). There was no abnormality in the hysteroscopy, but the patient underwent fascial hysterectomy with bilateral salpingo-oophorectomy, due to the extensive LEGH compromising the cone margin [1]. Her final histopathology report indicated a gastric-type mucinous AC of the cervix with moderate differentiation measuring $11 \mathrm{~mm}$ laterality and $6 \mathrm{~mm}$ in invasive depth.

In this case, the patient was operated on because of the LEGH diagnosis and gastric-type mucinous AC was found from the histopathology report. In addition, the tumor was small $(11 \times 6 \mathrm{~mm})$ and was moderately differentiated, while in our case the AC was large with low differentiation.

Our patient claimed that the previous screening for cervical cancer was at least 10 years ago. Cervical cancer screening is one of the best cancer prevention methods. However, there continue to be women who are not compliant with screening recommendations. Many die from this preventable cancer due to inadequate screening [11]. The early detection of cervical AC is very important, as is the need to differentiate it from endometrial cancer. According to the literature, the preoperative presence of glandular cells detected by cervical cytologic examination in patients with endometrial cancer is correlated with high-risk disease and might represent a predictor for local recurrence [12].

In both cases, patients underwent follow-up 3 months after surgery without clinical or imaging evidence of disease. Follow-up care after primary treatment should be conducted and coordinated by a physician experienced in the surveillance of cancer patients. A reasonable follow-up strategy involves follow-up visits every 3-4 months in the first 2 years, and every 6-12 months in years 3-5. At a minimum, follow-up visits should include a patient history and complete physical examination. However, the role of abdominal or pelvic CT, MRI or US as part of routine follow-up has not been fully evaluated in prospective studies [13].

Minimally invasive surgery (laparoscopic or robot-assisted radical hysterectomy) is also an option for women with early-stage cervical cancer [14]. According to the literature, minimally invasive radical hysterectomy is associated with no difference in oncologic outcomes as compared with the open approach. Tumor size $\geq 2 \mathrm{~cm}$ represents the most important risk factor influencing disease-free survival. However, patients with an IB1 tumor undergoing preoperative conization (vs cervical biopsy) are less likely to experience a recurrence [15]. Although minimally invasive surgery has many advantages for the patient, it was not feasible in our case.

\section{Conclusions}

Gastric-type mucinous AC of the cervix is a rare type of cancer with aggressive behavior. This type of malignancy is not related to high-risk HPV. The early diagnosis is very important, as this tumor is poorly symptomatic and very aggressive. When chemotherapy is not effective, radical surgery may be a solution for better survival.

\section{Disclosure}

The authors report no conflict of interest.

\section{References}

1. Lima PC, Teixeira J, Aires GN, Andrade LA. Endocervical gastric-type adenocarcinoma, an unrelated HPV tumour: difficulties in screening and diagnosis. BMJ Case Rep 2017; 2017: bcr2017219724.

2. Karamurzin YS, Kiyokawa T, Parkash V, et al. Gastric-type Endocervical Adenocarcinoma: An Aggressive Tumor With Unusual Metastatic Patterns and Poor Prognosis. Am J Surg Pathol 2015; 39: 1449-1457.

3. Kurman R, Carcangiu ML, Herrington CS, et al. WHO classification of tumours of female reproductive organs. IARC Press, Lyon 2014.

4. Hodgson A, Park KJ. Cervical adenocarcinomas: A heterogeneous group of tumors with variable etiologies and clinical outcomes. Arch Pathol Lab Med 2019; 143: 34-46.

5. Saida T, Sakata A, Tanaka YO, et al. Clinical and MRI characteristics of uterine cervical adenocarcinoma: Its variants and mimics. Korean J Radiol 2019; 20: 364-377.

6. Alvarado-Cabrero I, Patel P, Hoang L, et al. International Endocervical Adenocarcinoma Criteria and Classification (IECC). Am J Surg Pathol 2017; 42: 214-226.

7. Hodgson A, Park KJ, Djordjevic B, et al. International Endocervical Adenocarcinoma Criteria and Classification: Validation and Interobserver Reproducibility Anjelica. Am J Surg Pathol 2019; 43: 75-83.

8. Stolnicu S, Hoang L, Chiu D, et al. Clinical Outcomes of HPV-associated and Unassociated Endocervical Adenocarcinomas Categorized by the International Endocervical Adenocarcinoma Criteria and Classification (IECC). Am J Surg Pathol 2019; 43: 466-474.

9. Park CM, Koh HM, Park S, et al. Gastric type mucinous endocervical adenocarcinoma of the uterine cervix: very rare and interesting case. Obstet Gynecol Sci 2018; 61: 165-169.

10. Park KJ, Kiyokawa T, Soslow RA, et al. Unusual endocervical adenocarcinomas: an immunohistochemical analysis with molecular detection of human papillomavirus. Am J Surg Pathol 2011; 35: 633-646.

11. Benard VB, Thomas CC, King J, et al. Vital signs: cervical cancer incidence, mortality, and screening - United States, 2007-2012. MMWR Morb Mortal Wkly Rep 2014; 63: 1004-1009.

12. Casarin J, Bogani G, Serati M, et al. Presence of Glandular Cells at the Preoperative Cervical Cytology and Local Recurrence in Endometrial Cancer. Int J Gynecol Pathol 2019; doi: 10.1097/PGP.0000000000000642 [Epub ahead of print].

13. Elit L, Fyles AW, Oliver TK, et al. Follow-up for women after treatment for cervical cancer. Curr Oncol 2010; 17: 65-69.

14. Ramirez PT, Frumovitz M, Pareja R, et al. Minimally Invasive versus Abdominal Radical Hysterectomy for Cervical Cancer. N Engl J Med 2018; 379: 1895-1904.

15. Casarin J, Bogani G, Papadia A, et al. Preoperative Conization and Risk of Recurrence in Patients Undergoing Laparoscopic Radical Hysterectomy for Early Stage Cervical Cancer: A Multicenter Study. J Minim Invasive Gynecol 2020 Apr 19; S1553-4650(20)30190-4 [Epub ahead of print]. 